\title{
SOME PECULIARITIES IN LONGITUDE DISTRIBUTION OF SUNSPOT GROUPS OVER LAST FOUR ELEVEN-YEAR SOLAR CYCLES
}

\author{
A.L. Rybak \\ Central Astronomical Observatory of Pulkovo of RAS, Saint Petersburg, Russia, solarfish@alexeyryback.ru
}

This paper considers a longitude distribution of sunspot groups over 1982-2013, using data from the National Geophysical Data Center (Boulder, USA). The space-time distribution of sunspot groups is analyzed in coordinate sectors calculated from heliographic longitudes of the groups. A longitude extent of a coordinate sector is compared to the average size of one active region $\left(30-40^{\circ}\right)$. Then, in each coordinate sector, evolutionary activity of sunspot groups is summarized according to classification values after Malde throughout the observation period. The longitude distribution of large sunspot groups plotted in such a way does not reveal anticorrelation between Northern and Southern hemispheres in sunspot cycle 23.

Keywords: Sunspot cycle, active longitudes, classification values after Malde, coordinate sector, postdictors of active regions.

\section{INTRODUCTION}

The problem of nonuniform longitudinal distribution of solar activity (SA) over the Sun's surface has been the objective of much research since the late XIX century when the phenomenon was discovered [Wolfer, 1897]. Regular studies of the phenomenon of long-term excess of activity in separate longitude intervals, known as active longitudes, have been carried out at Pulkovo observatory since 1960s [Vitinsky, 1960; 1971]. According to the definition given by Yu.I. Vitinsky, a solar longitude interval, where SA is much higher than in other longitude intervals for a long time (several years), is called an active longitude. The ensemble of sunspots making up an active longitude is characterized by features that can differ from those of separate sunspots. So, for example, the rotational velocity of active longitudes is close to the Carrington one, whereas the rotational velocity of separate sunspots is governed by the differential rotation law. Previous studies [Ivanov, 1986; Plyusnina, 1992; Erofeev, 1997] have revealed that most sunspots over the course of an 11-year solar cycle are located inside formations revolving with periods close to 27 and 28 days.

The rotational velocity of active longitudes is now the subject of discussion. Kitchatinov and Olemskoi [Kitchatinov, Olemskoi, 2005] assert that in solar cycles 12-17 (1880-1944) the only active longitude distinguished with a sufficient degree of certainty was the interval with an ensemble of sunspots 
situated in the Northern Hemisphere in odd SA cycles. The rotation period of these active longitudes was slightly longer than 28 days. Plyusnina [Plyusnina, 2003] using the traditional definition of active longitudes and a more than 150-year series of sunspot observations shows that the sequence of bursts of activity observed in each solar cycle reveals a rotation period close to the Carrington one. These bursts of activity are spatially located inside formations with rotation periods close to 27 and 28 days. Qualitatively they represent the most powerful bursts of activity with modes of sunspot rotation with periods $~ 27-28$ days.

Active longitudes, as was stated above, can live for several solar cycles. As a minimum interval required to identify active longitudes it is wise to choose an SA cycle length of no less than 5-6 years. The currently most developed theory explaining solar activity with its intrinsic features of 11-year cyclicity and latitude variations in a sunspot zone during a cycle is the solar dynamo theory. Traditional axisymmetric approximations of the dynamo theory do not explain the longitude anisotropy in distribution of sunspots. The phenomenon of active longitudes was found in other solar-type stars too [Jetsu, 1996]. As in the Sun, active longitudes in stars are often spaced about $180^{\circ}$ apart. It has been noted that permanent active longitudes are alternatively active. This phenomenon discovered on stars was called flip-flop.

Active longitudes are explained using the notion of the relict magnetic field frozen in the Sun's radiant core and penetrating into the convective zone. Cowling [Cowling, 1945] was the first to recognize the possible existence of the relict magnetic field in the Sun's core. It has been noted that the time period of the ohmic attenuation of the large-scale field in the radiant zone is comparable to the age of the Sun. The most large-scale component of this field should still exist. Such ideas gained momentum in [Benevolenskaya et al., 1999; Kitchatinov et al., 2001]. According to [Kitchatinov et al., 2001], the relict magnetic field - an effect of the primary dynamo mechanism - was trapped from the ambient convective shell by the forming radiant core of the Sun at early stages of its evolution. The relict magnetic field is nonaxisymmetric, has a significant poloidal component, and its strength is a tenth of a gauss. During an 11-year sunspot cycle, the relict magnetic field is combined with the magnetic field that is generated by the solar dynamo. Due to the cycle-to-cycle magnetic polarity reversal according to Hale's Polarity Law, the resulting poloidal field can intensify or weaken. Consequently there emerges a toroidal field of different scales, and amplitudes of consecutive 11-year solar cycles have alternate heights [Mordvinov, Plyusnina, 2001].

This paper analyzes the longitude distribution of sunspot groups, using observations from the National Geophysical Data Center (NGDC, Boulder, USA). I show that the longitude distribution of sunspots depends on sizes of sunspots comprising the groups. The comprehensive study of the nonuniform longitudinal distribution of solar activity is of vital importance for prediction of geoeffectiveness of solar processes.

\section{OBSERVATIONAL MATERIAL}

Observations of sunspot groups were taken from the website of NGDC [ftp://ftp.ngdc.noaa.gov/STP/SOLAR_DATA/SUNSPOT_REGIONS]. The data are summaries from the worldwide network of solar observatories. The summaries are given in a final table that lists 
dates, universal time of observations, heliographic coordinates of sunspot groups, their areas in millionths of a solar hemisphere, linear size of the groups in longitude, the number of sunspots in a group, as well as Boulder class of groups according to McIntosh.

Due to the fact that the data have been collected based on observations made by the worldwide network of observatories, the final report represents a time-continuous data set that covers a period from December 1, 1981 to December 31, 2013.

I analyze peculiarities of longitude distribution of sunspot groups. For this purpose, I arrange all the observations in a heliographic longitude in chronological order. Then, I divide the resulting statistics for the Northern and Southern hemispheres into datasets with a fixed longitude interval (coordinate sectors). In each sector, I sum up sunspot indices of all the groups throughout the observation period of each of the groups. As a result, I obtain longitude-time series as a function of time $f(t)$ for further statistical analysis.

\section{BRIEF DESCRIPTION OF THE METHOD OF WAVELET-DECONVOLUTION OF LONGITUDE-TIME SERIES}

I minimize effects of rotational modulation in the data analysis, utilizing the method for waveletdeconvolution of the obtained longitude-time series [Mordvinov, Plyusnina, 2000].

To draw a longitude-time diagram from a longitude-time series, I perform wavelet filtration to isolate a component featuring the effect of rotational modulation caused by spatial inhomogeneity in sunspot regions. The wavelet filtration is done using the orthogonal wavelet expansion that is best suited to analyze non-stationary processes. As analyzing functions I have chosen Dobesh wavelets (Figure 1, $a$ ) [Astafyeva, 1996] that have convenient frequency-time location. Time scales of the wavelet functions used for the expansion are discrete and equal to powers of two. To analyze the longitude-time series as a function of time $f(t)$, I employ a discrete wavelet transform

$$
f(t)=\sum_{j, k=-\infty}^{\infty} c_{j k} \psi_{j k}(t),
$$

where $\psi_{j k}(t)$ are wavelet functions forming an orthonormal basis; wavelet-expansion coefficients are calculated from $c_{j k}=<f \psi_{j k}>$. The main effects of rotational modulation occur within 15-30 days. We should therefore perform the wavelet filtration, leaving during the inverse discrete wavelet transform only the coefficients that correspond to discrete scales of 8,16 , and 32 days. Then the filtered component representing the main effects of rotation takes the form

$$
f(t)=\sum_{j=3}^{j=5} \sum_{k=-\infty}^{\infty} c_{j k} \psi_{j k}(t)
$$

values of $2^{j}, j=3,4,5$ corresponding to the scales on which the rotational modulation occurs (Figure $1, b$ ). 


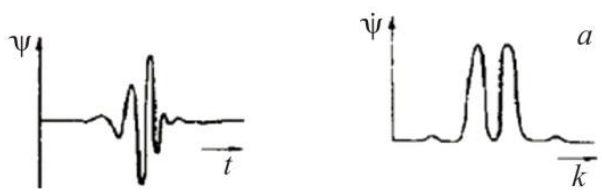

$b$

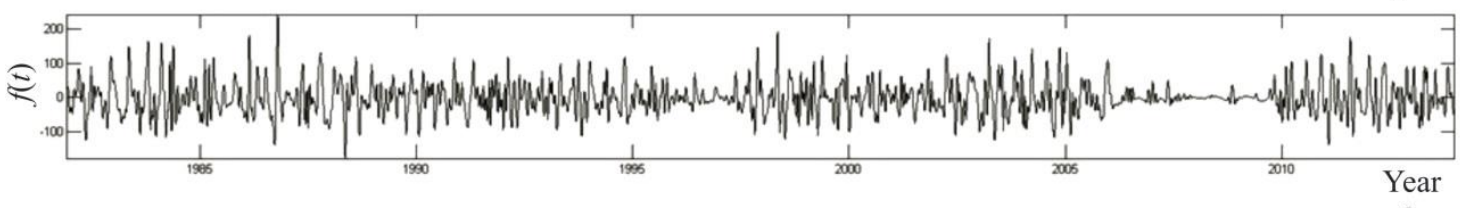

$c$

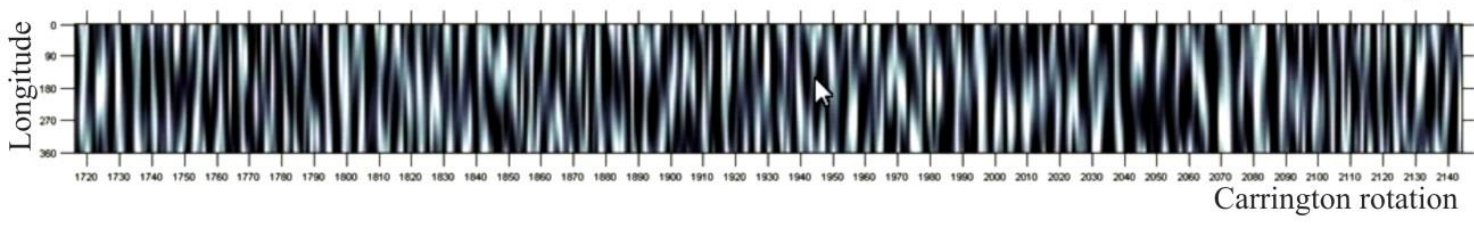

Figure 1. Dobesh wavelets (a), filtered dataset $(b)$, longitude-time diagram (c)

Then, I separate the filtered component into subarrays corresponding temporally to Carrington rotations and draw a rotation-to-rotation 2D gray-scale diagram (Figure 1,c). The observation period within a Carrington rotation corresponds to the heliographic longitude of the events. If a longitude time series is considered as convolution of distribution of sunspot groups over the solar disc, then the determination of spatial characteristics in time distribution of groups is an inverse process deconvolution of longitude-time series.

\section{COORDINATE SECTOR AS A MEASURE OF SUNSPOT ACTIVITY}

Sunspot groups have a typical longitudinal dimension depending on phase of their evolution. Sunspots appear on the Sun's surface as small single pores that have no considerable longitudinal extension. When developed, the sunspot groups become bipolar, growing in size. The typical longitudinal dimension of a bipolar sunspot group is $10-15^{\circ}$. In exceptional cases when a developed group is huge and has a complex multipolar configuration, its longitudinal dimension can exceed $20^{\circ}$.

Since I analyze longitude-time distribution of sunspot groups, I form further statistics according to the heliographic longitude of a current group, taking into account solar rotations from an initial time of observations. The resulting sequential statistics on longitudes is subdivided into sectors of fixed length with their sizes being comparable to linear sizes of an individual sunspot group and being not less than $0-15^{\circ}$ in longitude. Measure of power of each coordinate sector is the total evolutionary activity of all sunspot groups located in this sector during their passage through the solar disc according to the Boulder classification and its associated classification values after Malde [Malde, 1985].

Illustrate the above via examples of the longitude-time analysis of sequence of sunspot numbers in the Northern and Southern hemispheres obtained by averaging over 10, 20,30, and 40 . Curves of longitude distribution of sunspot groups are plotted by summing up the values for each solar rotation with the same longitude. For convenience, the resulting value can be normalized to the number of rotations 
used for the summation. Figure 2 shows the longitude distribution of groups with small sunspots. On the upper panel with $10^{\circ}$ averaging over the sector are multipeak curves. With increasing extent of the coordinate sectors it becomes apparent how the curves are smoothed; an obvious similarity exists between these distributions when sizes of the sectors are 30 and $40^{\circ}$.

Hence, plotting the coordinate series requires sectors of width no less than the average longitude extent of sunspot groups. We can obtain fairly consistent results by increasing the width of a coordinate sector to $30-40^{\circ}$. Further increase in this width results in absorption of two adjacent active longitudes. This problem lies in determining the active region.

According to [Vitinsky, 1966], the size of one active region on the Sun's surface is $30-40^{\circ}$; therefore, in further calculations I employ the coordinate sectors width of which varies within these limits.
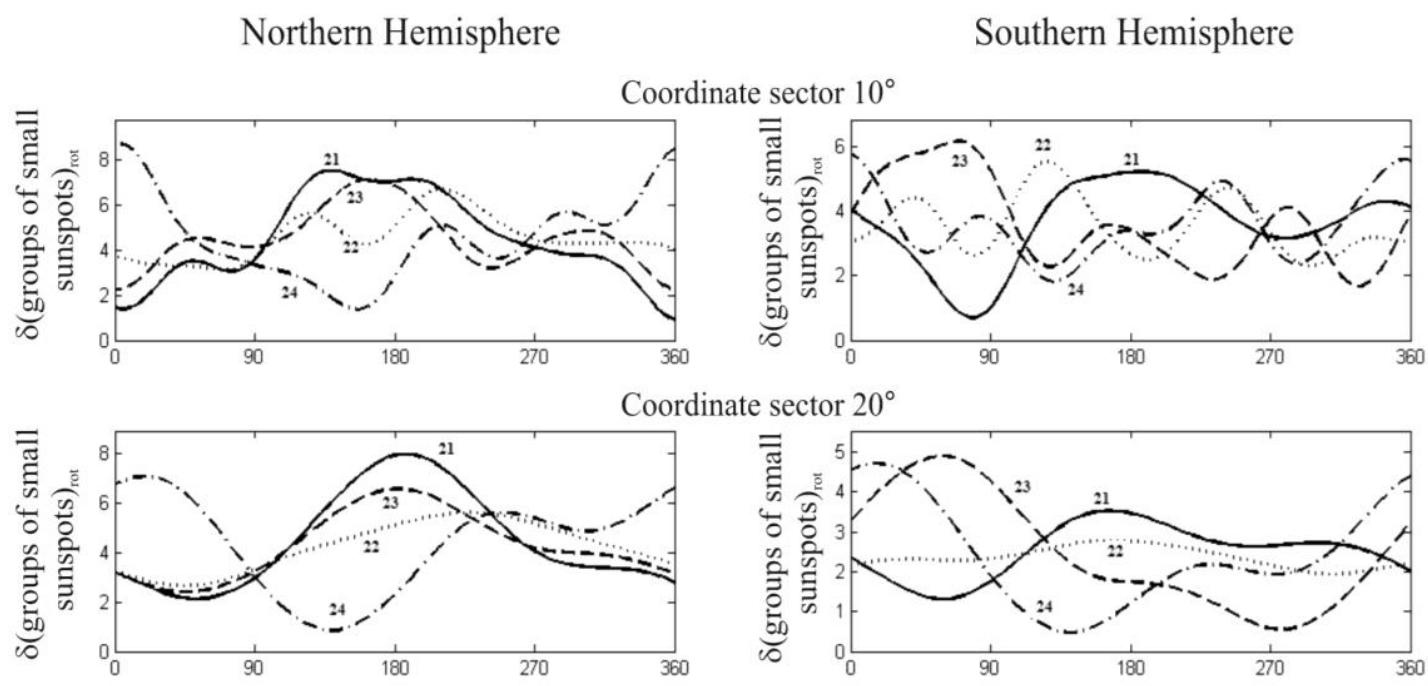

Coordinate sector $30^{\circ}$
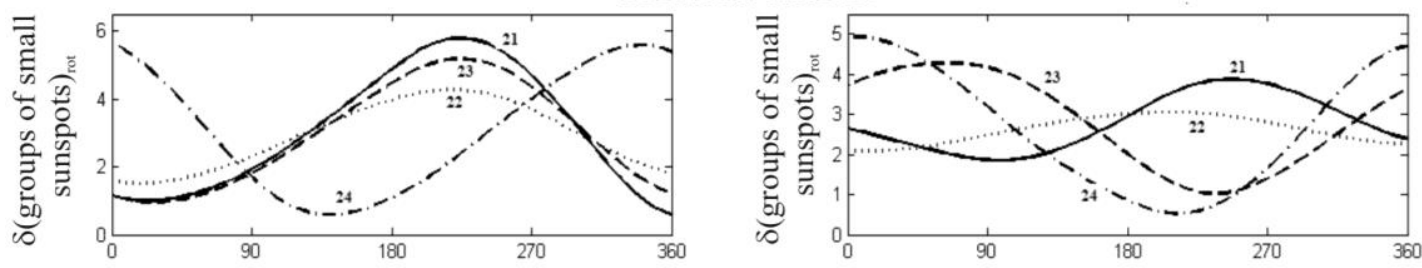

Coordinate sector $40^{\circ}$
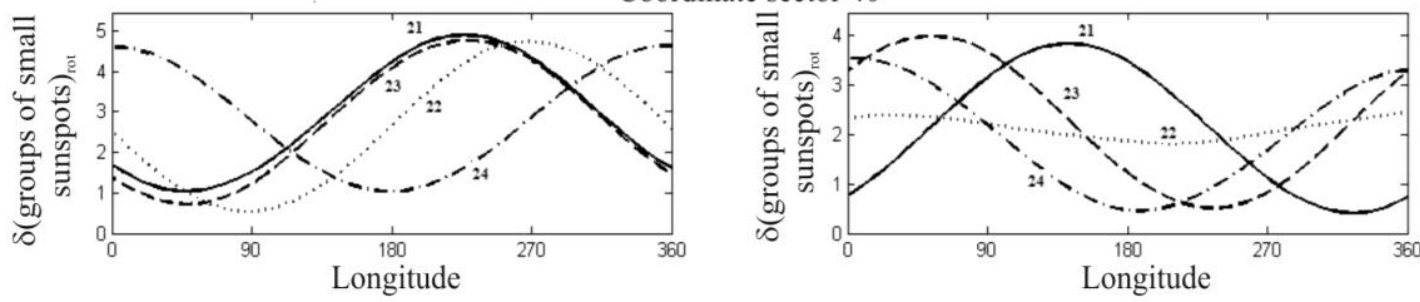

Figure 2. Selection of coordinate sector extent from longitude distribution of groups of small sunspots. Resulting distributions are obtained by summing up the normalized subarrays of the filtered component $f(t)$ of the respective longitude-time series over all Carrington rotations (Figure 1,c). Curves are marked with numbers of 11-year solar cycles. The extent of the coordinate sectors increasing, we can see smoothing and consistency of the obtained longitude distributions for each hemisphere. Besides, distributions of groups with small sunspots over the Northern Hemisphere in cycles 21 and 23 are nearly similar 
The analysis of the longitude distribution of small-sunspot groups, using the coordinate sector with maximum possible extent, has revealed that the small sunspots in the Northern Hemisphere during SA cycles 21 and 23 prevailed at the same longitudes with approximately equal level of concentration.

\section{LONGITUDE DISTRIBUTION OF GROUPS WITH SMALL AND LARGE SUNSPOTS}

Now consider how sunspots are distributed over longitudes depending on their sizes. Since the NGDC summaries contain the Boulder classification of the observed groups, I separate the entire sunspot statistics into small and large groups (see Table 1). The small groups are those with CV < 11 [Malde, 1985] (the Wolf number of one sunspot); the large ones $(\mathrm{CV}>36)$ are those containing at least one sunspot with a large penumbra according to the Boulder classification. All the rest of sunspot groups are taken to be intermediate (medium). Classification values after Malde (CV) allow us to account not only for the total area of a group but also sizes of the largest sunspots in this group. Therefore it would be more appropriate to specify sizes of the largest sunspots in these groups instead of sizes of the whole group: e.g., "groups of large sunspots" instead of "large groups of sunspots".

$\mathrm{CV}$ provide a more detailed description of evolution of sunspot groups than such sunspot indices as Wolf numbers and the total area of groups.

Let us consider, for one, a single sunspot with symmetric penumbra, which can be represented by two CV: $\mathrm{CV}=10$ and $\mathrm{CV}=40$. In both the former and latter cases, the Wolf numbers of such sunspots can be equal, and their total area rarely differs dramatically. From the viewpoint of evolutionary history of groups, sunspots with the Boulder classification HSX indicate the end of life of groups with ordinary bipolar configurations or of failed groups; whereas single sunspots of HHX class remind us of intense sunspot formation in this active region during several previous solar rotations.

Table 1. Classification values after Malde (CV) based on the evolutionary Boulder classification according to McIntosh [Malde, 1985].

\begin{tabular}{|c|c|c|c|c|c|c|c|c|c|c|c|}
\hline AXX & 1 & CSO & 11 & FAO & 21 & DAC & 31 & CHO & 41 & FHO & 51 \\
\hline BXO & 2 & CSI & 12 & DAI & 22 & EAC & 32 & CHI & 42 & DHI & 52 \\
\hline BXI & 3 & DRO & 13 & EAI & 23 & FAC & 33 & DKO & 43 & EHI & 53 \\
\hline HRX & 4 & ERO & 14 & FAI & 24 & DSC & 34 & EKO & 44 & FHI & 54 \\
\hline CRO & 5 & FRO & 15 & DSO & 25 & ESC & 35 & FKO & 45 & DKC & 55 \\
\hline CRI & 6 & DRI & 16 & ESO & 26 & FSC & 36 & DKI & 46 & EKC & 56 \\
\hline HAX & 7 & ERI & 17 & FSO & 27 & HKX & 37 & EKI & 47 & FKC & 57 \\
\hline CAO & 8 & FRI & 18 & DSI & 28 & CKO & 38 & FKI & 48 & DHC & 58 \\
\hline CAI & 9 & DAO & 19 & ESI & 29 & CKI & 39 & DHO & 49 & EHC & 59 \\
\hline HSX & 10 & EAO & 20 & FSI & 30 & HHX & 40 & EHO & 50 & FHC & 60 \\
\hline
\end{tabular}


Table 2. Average CV and their standard deviations calculated for groups of small, medium, and large sunspots for each of the 11-year solar cycles. In brackets is the total number of groups registered at stations of the network of NGDC observatories.

\begin{tabular}{|c|c|c|c|c|}
\hline $\begin{array}{c}\text { Solar cycle } \\
\text { (Carrington rotation) }\end{array}$ & $\begin{array}{c}\text { Groups of } \\
\text { small sunspots }\end{array}$ & $\begin{array}{c}\text { Groups of } \\
\text { medium sunspots }\end{array}$ & $\begin{array}{c}\text { Groups of } \\
\text { large sunspots }\end{array}$ & All sunspot groups \\
\hline $\begin{array}{l}\text { solar cycle } 21 \\
(\mathrm{CR} 1716-1781)\end{array}$ & $\begin{array}{l}4.51 \pm 3.58 \\
(17304)\end{array}$ & $18.38 \pm 5.96(9662)$ & $45.45 \pm 5.07(5558)$ & $\begin{array}{l}15.62 \pm 15.55 \\
(32524)\end{array}$ \\
\hline $\begin{array}{l}\text { solar cycle } 22 \\
(\mathrm{CR} 1782-1904)\end{array}$ & $\begin{array}{l}4.61 \pm 3.55 \\
(59822)\end{array}$ & $\begin{array}{l}18.89 \pm 5.47 \\
(32542)\end{array}$ & $\begin{array}{l}45.91 \pm 5.31 \\
(14976)\end{array}$ & $\begin{array}{l}14.70 \pm 14.77 \\
(107340)\end{array}$ \\
\hline $\begin{array}{l}\text { solar cycle } 23 \\
\text { (CR1905-2053) }\end{array}$ & $\begin{array}{l}5.45 \pm 3.67 \\
(33345)\end{array}$ & $\begin{array}{l}19.70 \pm 5.92 \\
(27619)\end{array}$ & $46.96 \pm 6.28(7682)$ & $\begin{array}{l}15.83 \pm 13.85 \\
(68646)\end{array}$ \\
\hline $\begin{array}{l}\text { solar cycle } 24 \\
(\mathrm{CR} 2054-2144)\end{array}$ & $\begin{array}{l}4.84 \pm 3.67 \\
(11973)\end{array}$ & $20.19 \pm 6.89(6565)$ & $46.26 \pm 6.99(2474)$ & $\begin{array}{l}14.51 \pm 14.50 \\
(21012)\end{array}$ \\
\hline $\begin{array}{l}\text { The entire observation } \\
\text { period }\end{array}$ & $\begin{array}{l}4.85 \pm 3.62 \\
(122444)\end{array}$ & $\begin{array}{l}19.23 \pm 5.86 \\
(76388)\end{array}$ & $\begin{array}{l}46.12 \pm 5.70 \\
(30690)\end{array}$ & $\begin{array}{l}15.15 \pm 14.60 \\
(229522)\end{array}$ \\
\hline
\end{tabular}

Similar reasonings are also valid for groups with open bipolar configurations, which have a large sunspot with symmetric penumbra. It is referred to groups of the Boulder classifications CHO, DHO, EHO, and FHO. Along with HHX-class large single sunspots, 5239 such groups have been detected under the NGDC program. The average CV $(43.72 \pm 4.44)$, the average number of sunspots in a group (6.54 \pm 7.10$)$, and the average total area of groups (278 \pm 207 millionths of a solar hemisphere) suggest that the first of the listed indices allows us to consider simple groups with a large symmetric sunspot as postdictors of the intense process of sunspot formation in previous solar rotations. Consequently, we can analyze the statistics on longitude-time distribution of groups of large sunspots, using average CV for such a sunspot class.

As for the ratio of CV to Wolf numbers, I in [Rybak, 2015] illustrate long-term cycle-to-cycle variations in average characteristics of sunspots.

Combining all observation data into the summary table, I obtain average CV characteristics which suggest satisfactory relationship between longitude irregularity and random statistical fluctuations. The average CV characteristics calculated for sunspots from $40^{\circ}$ coordinate sectors differ slightly from those given in Table 2 .

Longitude distribution of the sunspot groups is generally characterized by negative correlation of active longitudes in the Northern and Southern hemispheres. However, curves for sunspot cycle 23 show that groups with large sunspots in the Northern and Southern hemispheres predominated at the same longitudes (Figure 3). The comparison between longitude distributions of small, medium, and large sunspots over the Northern Hemisphere during solar cycle 23 suggests a considerable difference in the position of groups with large sunspots relative to other groups (the plot with a black arrow in Figure 3 ). 

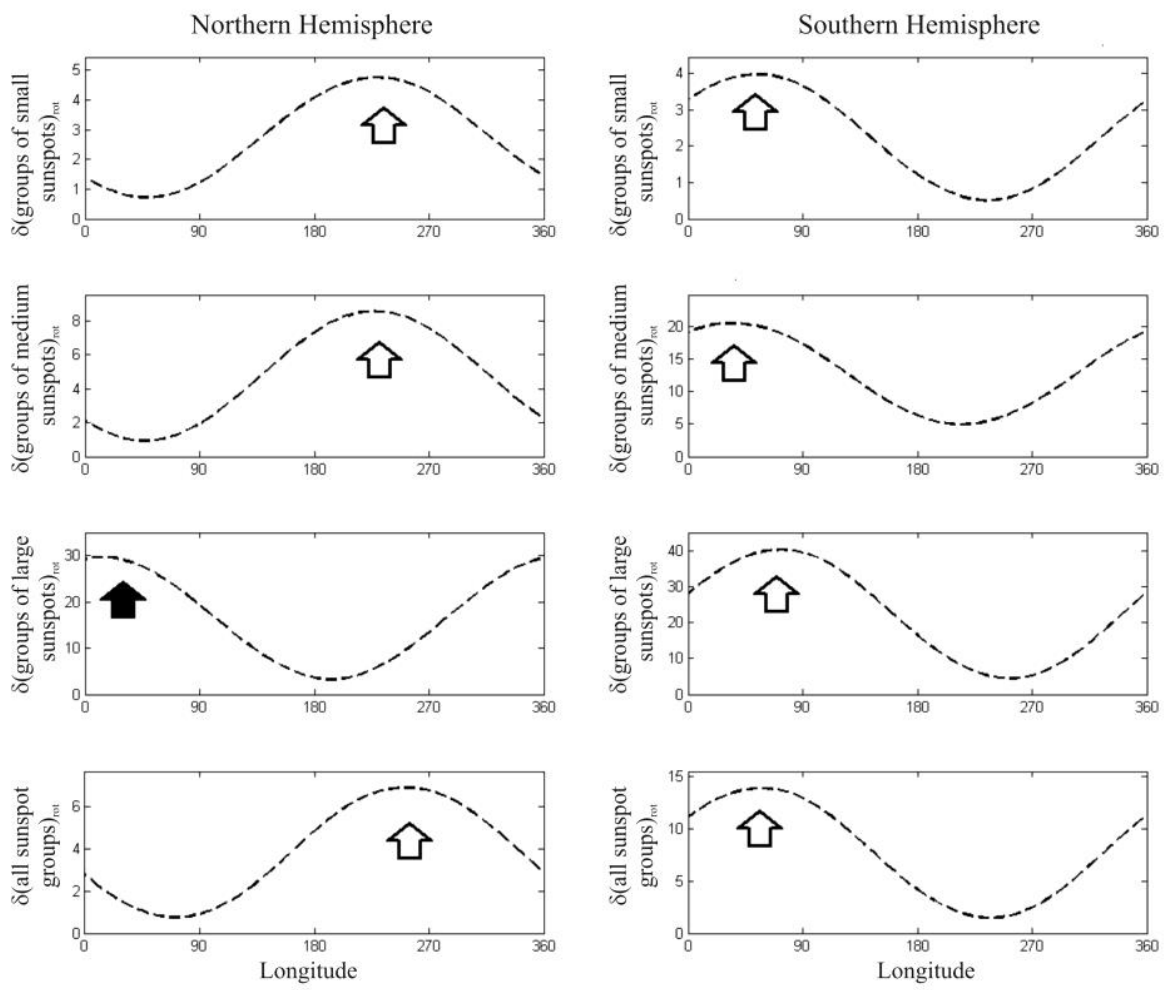

Figure 3. Similarity in longitude distribution of groups with large sunspots over the Northern and Southern hemispheres in SA cycle 23. During the 11-year solar cycle, large sunspots in the Northern and Southern hemispheres predominated in the longitude interval from 0 to $90^{\circ}$ (black arrow), whereas other sunspots in the Northern Hemisphere prevailed in the longitude interval from 180 to $270^{\circ}$. In the Southern Hemisphere, all the sunspot groups dominated in the longitude interval 0-90

\section{CONCLUSIONS}

1. I have demonstrated how effective it is to use coordinate sectors for studying active longitudes with a properly chosen scale of averaging over a heliographic longitude. This scale is $30-40^{\circ}$; it corresponds to the size of one active region [Vitinsky, 1966]. It is improper to further increase the size of the coordinate sector because this produces absorption of two adjacent active longitudes.

2. Using the coordinate sectors of sunspots, I found south-north asymmetry in the longitude distribution of sunspot groups. It is responsible for the negative correlation of curves for solar hemispheres. This may argue for the existence of an inclined nonaxisymmetric dipole with a rotation lasting approximately as long as the 11-year solar cycle.

3. Considering groups of small and large sunspots separately and from cycle to cycle yields the following objective laws:

a) proximity of active longitudes in the Northern Hemisphere for small sunspots in SA cycles 21 and 23;

b) similarity in distribution of active longitudes over the Northern and Southern hemispheres for groups with large sunspots in solar cycle 23; 
c) a significant difference in longitude distribution of groups with small and large sunspots over the Northern Hemisphere in cycle 23, which shows that in that cycle the predominance of groups with large sunspots in the Northern Hemisphere at heliographic longitudes dropped out of the common pattern. This circumstance may be one of the causes of the relative increase in the number of small sunspots during SA cycles 23 and 24.

\section{REFERENCES}

Astaf'eva N.M. Wavelet analysis: Theory grounds and examples of applications. Uspekhi fizicheskikh nauk. [Physics-Uspekhi (Advances in Physical Sciences)]. 1996, vol. 166, iss. 11, pp. 1145-1170. (In Russian).

Benevolenskaya E.E., Hoeksema J.T., Kosovichev A.G., Scherrer P.H. The interaction of new and old magnetic fluxes at the beginning of solar cycle 23. The Astrophys. J. 1999, vol. 517, iss. 2, pp. 163-166. DOI: 10.1086/312046.

Cowling T.G. On the Sun's general magnetic field. Monthly Notices of the Royal Astronomical Society. 1945, vol. 105, p. 166. DOI: 10.1093/mnras/105.3.166.

Erofeev D.V. The relationship between kinematics and spatial structure of the large-scale solar magnetic field. Solar Phys. 1997, vol. 175, iss. 1, pp. 45-58. DOI: 10.1023/ A:1004981317538.

Ivanov E.V. On space-time distribution of faculae and sunspots. Solnechnye dannye. Byul. [Solar Data Bulletin] 1986, no. 7, p. 61. (In Russian).

Jetsu L., Pohjolainen S., Pelt J., Tuominen I. Is the longitudinal distribution of solar flares nonuniform? Astron. Astrophys. 1996, vol. 318, pp. 293-307.

Kitchatinov L.L., Olemskoi S.V. Active longitudes of the sun: the rotation period and statistical significance. Pis'ma $v$ Astronomicheskii Zhurnal [Astron. Lett.]. 2005, vol. 31, iss. 4, pp. 280-284. DOI: 10.1134/1. 1896072. (In Russian).

Kitchatinov L.L., Jardine M., Collier Cameron A. Pre-main sequence dynamos and relic magnetic fields of solartype stars. Astron. Astrophys. 2001, vol. 374, pp. 250-258. DOI: 10.1051/ 0004-6361:20010729.

Malde K.I. „Klassifikationswerte“, eine neue Messung der Sonnenaktivitat? Sonne. Jahrg. 9. 1985, no. 36, pp. 159-163.

Mordvinov A.V., Plyusnina L.A. Cyclic changes in solar rotation inferred from temporal changes in the mean magnetic field. Solar Phys. 2000, vol. 197, iss. 1, pp. 1-9. DOI: 10.1023/A:1026559227059.

Mordvinov A.V., Plyusnina L.A. Coherent structures in the dynamics of the large-scale solar magnetic field. Astronomicheskii Zhurnal [Astron. Rep.]. 2001, vol. 45, iss. 8, pp. 652-658. DOI: 10.1134/ 1.1388930. (In Russian).

Plyusnina L.A. Two-level structure of elements of the Sun's large-scale magnetic field rotation. Issledovaniya po geomagnetizmu, aeronomii i fizike Solntsa [Res. on Geomagnetism, Aeronomy and Solar Physics]. Novosibirsk: Nauka.1992, vol. 99, pp. 138-144. (In Russian).

Plyusnina L.A. Climatic and ecological aspects of solar activity. Trudy VII Pulkovskoi mezhdunarodnoi konferentsii po fizike Solntsa [Proc. VII Pulkovo International Conference on Solar Physics]. Saint Petersburg, 2003, p. 353. (In Russian).

Rybak, A.L. The Malde classification index and long-term changes in average sunspots features. Geomagnetism and Aeronomy. 2015, vol. 55, iss. 7. pp. 884-886. DOI: 10.1134/S00 16793215070208.

Vitinskii Yu.I. On the peculiarities in longitudinal distribution of solar activity. Izvestiya GAO RAN [Trans. of the Pulkovo Observatory of RAS]. 1960, no. 163, pp. 96-105. (In Russian).

Vitinskii Yu.I. Solar activity morphology. Moscow, Nauka Publ., 1966, p. 199. (In Russian).

Vitinskii, Yu. I. Centers of activity and their active longitudes. Izvestiia GAO RAN [Trans. of the Pulkovo Observatory of RAS]. 1971, no. 189-190. pp. 10-22. (In Russian).

Wolfer A. Publ. Sternv. Eidg. Polytechn. Zurich, 1897, vol. 1, p. 1.

URL:ftp://ftp.ngdc.noaa.gov/STP/SOLAR_DATA/SUNSPOT_REGIONS. 